\title{
Phytoprotection
}

phytoprotection

\section{Liste des arbitres 1990-1991}

List of reviewers 1990-1991

Volume 73, numéro 2, 1992

URI : https://id.erudit.org/iderudit/706024ar

DOI : https://doi.org/10.7202/706024ar

Aller au sommaire du numéro

Éditeur(s)

Société de protection des plantes du Québec (SPPQ)l

ISSN

0031-9511 (imprimé)

1710-1603 (numérique)

Découvrir la revue

Citer ce document

(1992). Liste des arbitres 1990-1991. Phytoprotection, 73(2), 83-84.

https://doi.org/10.7202/706024ar d'utilisation que vous pouvez consulter en ligne.

https://apropos.erudit.org/fr/usagers/politique-dutilisation/ 
Liste des arbitres 1990-1991

List of reviewers 1990-1991

Asselin, A.

Bélanger, $\mathrm{R}$.

Benhamou, N.

Boiteau, G.

Bourgeois, G.

Carling, D.E.

Comeau, A.

Copeman, R.J.

Coulman, B.

Cunningham, J.C.

Devaux, A.

Dostaler, D.

Doster, M.A.

Doyon, D.

Duchesne, R.-M.

Dumas, E.

Evans, D.

Glazener, J.

Hall, R.

Hamilton, R.I.

Hébert, $C$.

Holliday, N.J.

Hsiao, T.H.

Hubbes, M.

Hume, D.J.

Ivany, J.A.

Johnson, S.G.

Kimpinski, J.

Laliberté, J.F.

Lee, R.E.

Leroux, G.D.

Nigam C.

Otrysko, B.E.

Parent, L.-E.

Patrick, Z.A.

Paulitz, T.
Université Laval, Québec (Québec)

Université Laval, Québec (Québec)

Université Laval, Québec (Québec)

Agriculture Canada, Fredericton (New Brunswick)

Agriculture Canada, Saint-Jean-sur-Richelieu (Québec)

University of Alaska, Palmer (Alaska)

Agriculture Canada, Sainte-Foy (Québec)

University of British Columbia, Vancouver (British Columbia)

McGill University, Sainte-Anne-de-Bellevue (Québec)

Forêts Canada, Sault-Sainte-Marie (Ontario)

MAPAQ, Saint-Hyacinthe (Québec)

Université Laval, Québec (Québec)

Cornell University, Ithaca (New York)

MAPAQ, Sainte-Foy (Québec)

MAPAQ, Sainte-Foy (Québec)

Institut National de Recherche Agronomique, Dijon, France

Department of Biology, Kalamazoo College, Kalamazoo (Michigan)

USDA-ARS, Beltsville (Maryland)

University of Guelph, Guelph (Ontario)

Agriculture Canada, Vancouver (British Columbia)

Centre de foresterie des Laurentides, Forêts Canada, Sainte-Foy (Québec)

University of Manitoba, Winnipeg (Manitoba)

Department of Biology, Utah State University, Logan (Utah)

University of Toronto, Toronto (Ontario)

University of Guelph, Guelph (Ontario)

Agriculture Canada, Charlottetown (Prince Edward Island)

Université Laval, Québec (Québec)

Agriculture Canada, Charlottetown (Prince Edward Island)

Institut Armand Frappier, Ville de Laval (Québec)

Miami University, Hamilton (Ohio)

Université Laval, Québec (Québec)

Forestry Canada, Maritime Forestry Center, Fredericton (New Brunswick)

MAPAQ, Les Buissons (Québec)

Université Laval, Québec (Québec)

University of Toronto, Toronto (Ontario)

McGill University, Sainte-Anne-de-Bellevue (Québec) 
Pelletier, Y.

Peterson, J.F.

Reeleder, R.D.

Rennie, R.J.

Retnakaran, A.

Sallé, G.

Simard, R.

Smith, D.

Sutton, J.C.

Swanton, $\mathrm{C}$.

Tartier, L.

Tekauz, A.

Therrien, $P$.

Vernon, R.

Vrain, T.C.

Wright, N.S.
Agriculture Canada, Fredericton (New Brunswick)

McGill University, Sainte-Anne-de-Bellevue (Québec)

McGill University, Sainte-Anne-de-Bellevue (Québec)

Agriculture Canada, Lethbridge (Alberta)

Forestry Pest Management Institute, Sault-Ste-Marie (Ontario)

Université Pierre et Marie Curie, Paris, France

Agriculture Canada, Sainte-Foy (Québec)

McGill University, Sainte-Anne-de-Bellevue (Québec)

University of Guelph, Guelph (Ontario)

University of Guelph, Guelph (Ontario)

MAPAQ, Saint-Hyacinthe (Québec)

Agriculture Canada, Winnipeg (Manitoba)

Ministère de l'Énergie et des Ressources, Québec (Québec)

Agriculture Canada, Vancouver (British Columbia)

Agriculture Canada, Vancouver (British Columbia)

Agriculture Canada, Vancouver (British Columbia) 